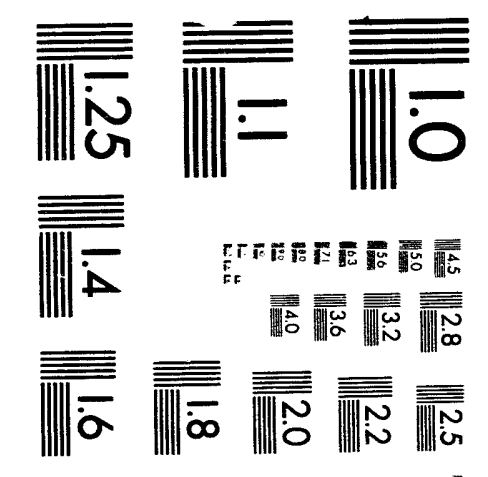



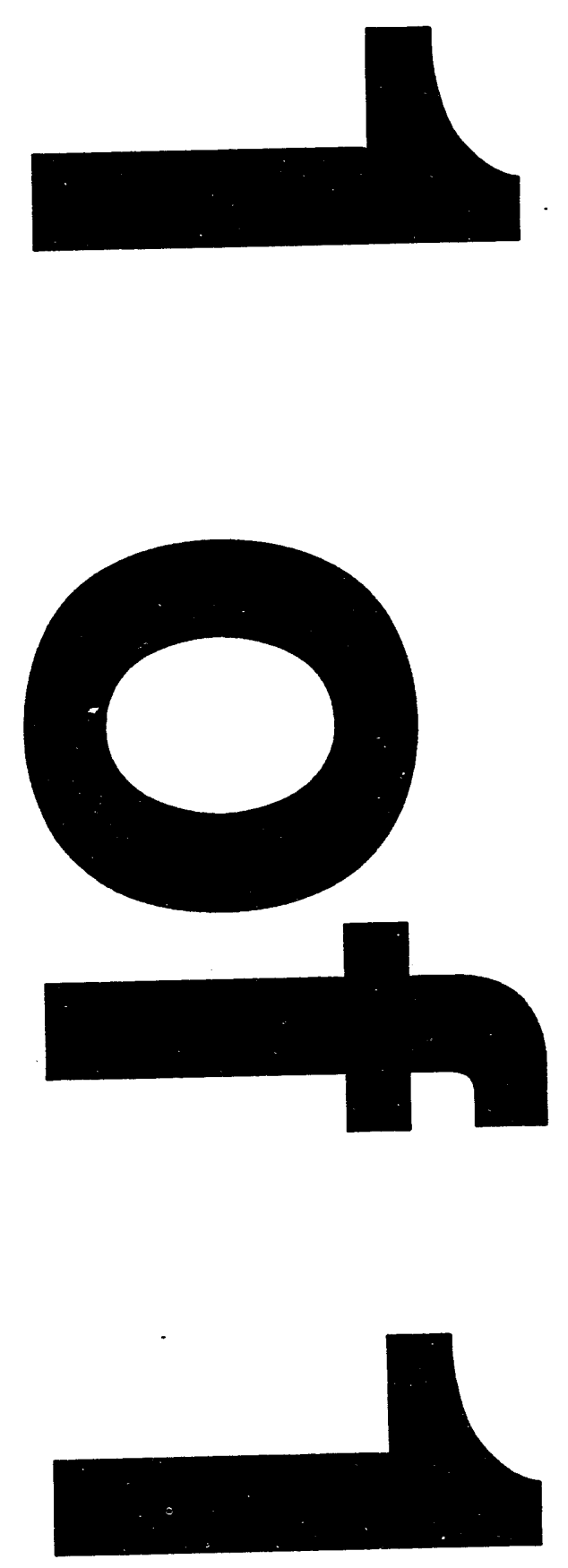
- LA-UR 93-3914

Conf-9310235 -.

DEC 131933

Los Alamos National Laboratory is operated by the University of California for the United States Department of Energy under chotraty-140-ENG-36

TITLE: $\quad$ A SURVEY OF OPEN PROBLEMS IN SYMPLECTIC INTEGRATION

AUTHOR(S): $\quad$ Robert I. McLachlan and Clint Scovel

SUBMITTED TO: $\quad$ Proc. of International Symposium on

Integration Algorithms for Classical Mechanics

The Fields Institute

Ontario, Canada

October 14-17, 1993

\section{DISCLAIMER}

This report was prepared as an account of work sponsored by an agency of the United States Government. Neither the United States Government nor any agency thereof, nor any of their employees, makes any warranty, express or implied, or assumes any legal liability or responsibility for the accuracy, completeness, or usefulness of any information, apparatus, prcduct, or process disclesed, or represents that its use would not infringe privately owned rights. Reference herein to any specific commercial product, process, or service by trade name, trademark, manufacturer, or otherwise does not necessarily constitute or imply its endorsement, recommendation, or favoring by the United States Government or any agency thereof. The views and opinions of authors expressed herein do not necessarily state or reflect those of the United States Government or any agency thereof.

By acceptance of this article, the publisher recognizes that the U.S. Government retains a nonexclusive royalty-free license to publish or reproduce the published form of this contribution or to allow others to do so, for U.S. Government purposes.

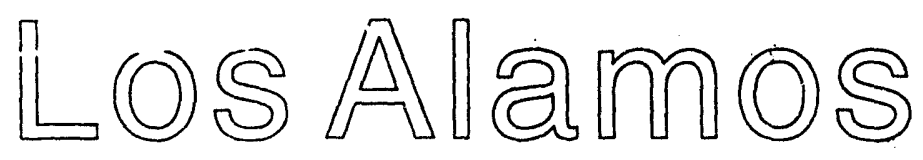

\section{Los Alamos National Laboratory Los Alamos New Mexico 87545}




\title{
A Survey of Open Problems in Symplectic Integration
}

\author{
Robert I. McLachlan ${ }^{1}$ and Clint Scovel ${ }^{2}$ \\ 1 Program in Applied Mathematics \\ University of Colorado at Boulder \\ Boulder CO 80309-0526 (rxm@boulder.colorado.edu) \\ 2 Computer Research Group, C-3, MS-B265 \\ Los Alamos National Laboratory, \\ Los Alamos, NM 87545 (jcs@cabron.c3.lanl.gov) \\ October 15,1993
}

\section{Dedicated to Feng Kang.}

\begin{abstract}
We present a personal view of the most important open problems and areas for research in symplectic integration and in the more general study of geometric structure in numerical methods. At present, this survey is incomplete, but we hope with the help of our colleagues to be able to include in the proceedings of this conference a more comprehensive survey.
\end{abstract}

\section{Table of Contents}

1 Introduction . . . . . . . . . . . . . . . . 1

2 Composition methods . . . . . . . . . . . . . . . . . . . . 2

2.1 High-order methods .................. 2

2.2 Negative time steps . . . . . . . . . . . . . . . . . . . . . . . . . . . . . . . .

2.3 Existence of splittings . . . . . . . . . . . . . . . . . . . . . . . . . . . . . . . . . .

2.4 Symplecticity for free . . . . . . . . . . . . . . . 4

3 Multiderivative methods . . . . . . . . . . . . . . . . 4

4 No-derivative methods . . . . . . . . . . . . . . 5

5 Implicit methods . . . . . . . . . . . . . . . . . . . . 5

6 Generating functions . . . . . . . . . . . . . . . 5

7 Multistep methods . . . . . . . . . . . . . . . . 6

8 Preservation of integrals . . . . . . . . . . . . . . . . . . . . 8

9 Variable time steps . . . . . . . . . . . . . . . . . . . . . . . 8

10 Constrained systems . . . . . . . . . . . . . . . . . . 9

10.1 Dirac constraints . . . . . . . . . . . . . . . . . . . . . . . . . . . . . .

10.2 Fewer implicit steps . . . . . . . . . . . . . . . . . . . . . . . . . . . . . . . 10

10.3 Generating functions . . . . . . . . . . . . . . . . . 10

10.4 Automatic preservation of quadratic constraints . . . . . . . 11

11 Approximation of infinite-dimensional Poisson manifolds . . . 11 
11.1 The sine bracket . . . . . . . . . . . . . . . . 13

11.2 Moment truncations ... . . . . . . . . . . . . . 13

11.3 Other approaches ................... 14

11.4 Augmentation and decoupling of semi-direct products . . . . . 15

12 Theoretical performance . . . . . . . . . . . . . . 16

12.1 The KAM theorem . . . . . . . . . . . . . . 16

12.2 Extensions of Hamiltonian stability theorems . . . . . . . . . 17

12.3 Reversible adiabatic invariance theorem . . . . . . . . . 18

12.4 Chaos ....................... 18

13 Dissipative systems . . . . . . . . . . . . . . . 18

14 Assorted issues ...................... . . . 20

14.1 The discrete Lagrangian method . . . . . . . . . . . . . . . 20

14.2 Reversible systems . . . . . . . . . . . . . . . . . . 20

14.3 Volume preservation ... . . . . . . . . . . . 20

14.4 Symplectic lattice maps ... . . . . . . . . . . . . 21

14.5 Standard methods .................... . . 21

14.6 Shooting methods ... . . . . . . . . . . . . . 21

14.7 Elliptic equations . . . . . . . . . . . . . . . 21

\section{Introduction}

In the past few years there has been a substantial amount of research on symplectic integration. The subject is only part of a program concerned with numerically preserving a system's inherent geometrical structures. Volume preservation, reversibility, local conservation laws for elliptic equations, and systems with integral invariants are but a few examples of such invariant structures. In many cases one requires a numerical method to stay in the smallest possible appropriate group of phase space maps. It is not the authors' opinion that symplecticity, for example, automatically makes a numerical method superior to all others, but it is our opinion that it should be taken seriously and that a conscious, informed decision be made in that regard.

We present here a survey of open problems in symplectic integration, including other problems from the larger program. This is not intended as a review of symplectic integration (see $[111,110,131]$ ) and is naturally derived from the authors' own research interests. At present, this survey is incomplete, but we hope with the help of our colleagues to be able to include in the proceedings of this conference a more comprehensive survey.

Many of the problems mentioned here call for numerical experimentation, some for application of suggested but untested methods, some for new methods, and some for theorems. Some envisage large research programs. We hope that this survey helps to communicate the outstanding problems in this rapidly growing field to the research community.

\section{Composition methods}

\subsection{High-order methods}

It is now a standard practice in structure-preserving integration to compose one or more low-order methods (usually first or second order) with appropriately 
chosen time steps to obtain a higher-order method. If one has a differential equation $\dot{x}=X(x)=A(x)+B(x)$ such that $\dot{x}=A$ and $\dot{x}=B$ can both be solved analytically, then one may use the compositions

$$
\ldots \exp \left(a_{2} t A\right) \exp \left(b_{1} t B\right) \exp \left(a_{1} t A\right)
$$

or

$$
\ldots S\left(w_{2} t\right) S\left(w_{1} t\right)
$$

where $S(t)=\exp \left(\frac{1}{2} t A\right) \exp (t B) \exp \left(\frac{1}{2} t A\right)$ is the leapfrog method. Forest et al. [36] have noted that $S$ just needs to be symmetric, i.e., $S^{-1}(t)=S(-t)$. One may expand these compositions as $\exp \left(\sum_{i=1}^{\infty} t^{i} X_{i}\right)$ where $X_{i} \in L^{n}(A, B)$, the space spanned by Lie brackets of $A$ and $B$ of length $n$. This space is a subspace of the free Lie algebra generated by $A$ and $B[11,101]$ whose dimension is known. Thus there are $\sum_{n=1}^{p} \operatorname{dim} L^{n}(A, B)$ determining equations which must be satisfied for a method to have order at least $p$. It is traditional to compare different solutions of the determining equations based on an effective error constant-some measure of the first term in the local truncation error at constant work.

Sometimes, breaking a symmetry is not obviously compensated for by the increase in the number of free parameters [78]:

- Is there a symmetric sixth-order method which is better than those composed of symmetric steps?

A special case is when $H=H_{A}(p)+H_{B}(q)$ where $H_{A}$ is quadratic in $p$. In that case $[B,[B,[B, A]]] \equiv\left[B^{3} A\right]$ is identically zero (here $A$ is the Hamiltonian vector field corresponding to the Hamiltonian $H_{A}$, etc.) and the determining equations corresponding to this term and its Lie brackets may be dropped. This leads to a definite advantage for nonsymmetric fourth-order and for symmetric sixth-order methods.

- Is there a symmetric eighth-order Runge-Kutta-Nyström method which is better than general methods of type (2)?

However, there are zero brackets of $H_{A}$ and $H_{B}$ which are not implied solely by the condition $\left[B^{3} A\right]=0$. This leads to the following questions:

- Describe the Lie algebra generated by the vector fields $A$ and $B$ when $\left[B^{3} A\right]=0$.

- Describe the Lie algebra generated by the Hamiltonian functions $p^{2}$ and $H_{B}(q)$.

There are no differences between these two cases for $n \leq 7$, so the popular Runge-Kutta-Nyström methods of order 7 or less (or 10 if symmetric, see [78]) apply in the more general case $\left[B^{3} A\right]=0$, which has some applications in nonlinear wave equations [77]. Note, however, that the eighth-order term $\left[B^{2} A,\left[B^{2} A, B^{1} A\right]\right]$ is zero under the $p^{2}+H_{B}(q)$ splitting, but is not explicitly a bracket of $\left[B^{3} A\right]$.

- Are there any other useful special cases, or different kinds of compositions, which lead to better methods? 


\subsection{Negative time steps}

Skeel [116] has raised the following question, which has applications in the case that $A$ is Hamiltonian but $B$ is not, such as in discretizations of reaction-diffusion equations. Because of the weight of the available evidence we give it as a conjecture.

- Prove that there are no composition methods of the form (1) of order higher than two in which all the substeps $\exp \left(b_{i} t B\right)$ have $b_{i}>0$.

For order two, of course, leapfrog suffices. Goldman and Kaper [45] solved the six-stage third-order determining equations explicitly; none of the solutions had all the $b_{i}>0$. Sheng [114] and Suzuki [122] have proved that at least one of the $a_{i}$ or the $b_{i}$ must be negative for any order greater than two, For Hamiltonian systems there is no evidence that negative substeps are detrimental, although naturally large substeps lead to large truncation errors. For a discussion of splitting methods for parabolic systems see Glasner [44].

\subsection{Existence of splittings}

What is special about the class of Hamiltonian systems-or of all vector fieldswhich possess splittings $X=\sum_{i} X_{i}$ for which the pieces can be integrated exactly? Even non-canonical systems( and non-Hamiltonian systems) are surprisingly amenable to this approach, such as Lie-Poisson systems in which the Hamiltonian only couples variables within the Lie algebra's abelian subalgebras [76]. This class includes, for example, the moment algebra with separable energy function, the rigid body, and the sine-bracket truncation of the 2D Euler equations (see $\S 11.1$ ).

For most splittings used in practice, the pieces $X_{i}$ are either linear or have shear flows, such as when $H=H_{A}(p)+H_{B}(q)$. One can leave this class and ask,

- which Hamiltonian vector fields $X$ can be written as $X=\sum_{i=1}^{n} X_{i}$ where each $X_{i}$ is completely integrable?

There is a huge arsenal of such vector fields to call on, for both canonical [96] and noncanonical $[26,35]$ systems. There is also the freedom to change variables in $X$ before splitting. In this problem one is supposed to start with $X$, not with the $X_{i}$ !

Naturally one does not want $n$ to be too large. For example, for any Euler equation (a Lie-Poisson system with quadratic Hamiltonian), the Hamiltonian may be diagonalized and each term integrated [100]. This leads to an $\mathcal{O}\left(N^{5}\right)$ algorithm for the $N \times N$ sine-bracket truncation of the 2D Euler fluid equations (see $§ 11.1)$, as opposed to the $\mathcal{O}\left(N^{3} \log N\right)$ possible with a different splitting [76].

Nor should $\exp \left(t X_{i}\right)$ be too difficult to evaluate-there is a big difference between proving a system integrable and actually evaluating its solution. For example, the Hamiltonian for the motion of $n$ point vortices is $H=\sum_{i<j} V\left(x_{i}, x_{j}\right)$, where vortex $i$ is located at $x_{i} \in \mathbb{R}^{2}$. The two-vortex interaction term $V\left(x_{1}, x_{2}\right)=$ $-\frac{1}{4 \pi} \log \left|x_{1}-x_{2}\right|$ has a simple flow (the vortices merely rotate about their center of vorticity in appropriate coordinates [104]), leading to an explicit symplectic 
integrator of any order for this problem. It is probably not efficient to use the integrability of the three-vortex problem. Another non-artificial example is that of Wisdom and Holman [130], who successfully integrated the $n$-planet solar system with such a method. In Jacobi coordinates, they wrote $H=K(q, p)+V(q)$ where $K$ represents $n$ Sun-planet two-body Kepler problems (all mutually commuting, and easily solved) and $V$ represents the $n(n-1) / 2$ planet-planet interaction terms, whose flow is a shear. This splitting is much more accurate for this problem than the trivial $H_{A}(p)+H_{B}(q)$ splitting. However, it is only accurate for solar system-like configurations; it is not uniformly accurate in the planet-planet distances. We therefore pose the problem:

- Develop an explicit symplectic integrator for the Newtonian $n$-body problem which is uniformly accurate near two-body collisions.

(Three body collisions not being well-posed, we can't be expected to deal with.) A promising approach is to use a partition of unity on the Hamiltonian so that each close pair is integrated exactly, with smooth transitions in the splitting as pairs break up. Unfortunately the $n$ kinetic energy terms do not apportion easily amongst the $n(n-1) / 2$ potential energy terms.

\subsection{Symplecticity for free}

Take a differential equation $\dot{x}=X(x)$. Let $\varphi(t)=1+t X$ be Euler's method, which is not symplectic. It has been noticed independently by Iserles and by Sanz-Serna (unpublished), that $\varphi(t / 2) \varphi^{-1}(-t / 2)$ is the midpoint rule, $x^{\prime}=$ $x+t X\left(\left(x+x^{\prime}\right) / 2\right)$, which explains the good long-time behavior of the similar but nonsymplectic composition $\varphi^{-1}(-t / 2) \varphi(t / 2)$, which is second-order AdamsMoulton: $x^{\prime}=x+t\left(X(x)+X\left(x^{\prime}\right)\right) / 2$. Generalizing this cute example would give a huge new class of symplectic integrators.

- Give a necessary and sufficient condition on $\varphi(t)$ such that $\varphi(t / 2) \varphi^{-1}(-t / 2)$ is symplectic.

\section{Multiderivative methods}

For some systems, such as those of molecular dynamics, the incremental cost of evaluating the derivative of the forces is small [116]. Therefore one should

- Investigate all possible ways of including extra derivative information in a symplectic integrator.

In fact, the earliest symplectic integrators based on the Taylor series solution of the Hamiltonian-Jacobi equation $[31,18]$, did use derivatives, but these methods are not considered competitive today. An exception is the Stoffer ansatz $[120,111]$, which achieves fourth order in one stage using only the second derivative of the Hamiltonian. This may allow one to use larger time steps than in the high-order force-only composition methods. There are also explicit multiderivative symplectic schemes $[79,65]$. Unfortunately, linear multiderivative symplectic schemes do not exist [47], and indeed, all of the above examples are nonlinear in the derivatives. 


\section{No-derivative methods}

Sometimes the Hamiltonian is so complicated that one does not wish to calculate its derivative by hand, or even by a symbolic manipulator.

- How efficient can symplectic integrators be, given only the Hamiltonian function?

One approach would be to form the vector field using automatic differentiation, which is now a mature technique [61,33]. Another is to use a fixed lattice in phase space to define an interpolated piecewise-polynomial Hamiltonian. A symplectic map $f(x)$ can be defined by taking the lattice points nearest $x$ and computing using the vector field corresponding to that piece of the interpolating Hamiltonian. (Or can one finite-difference the Hamiltonian about $x$ ? - - see $\S 12.2$.) There is no cost associated with making the interpolation error as small as desired, but care must be taken to make the $f(x)$ as smooth as possible, so that, e.g., KAM theory can apply.

\section{Implicit methods}

Some vector fields, alas, cannot be split. Then one has the generating function and Gauss-Legendre Runge-Kutta methods. Note that as the order increases, the latter do not suffer combinatorial explosion as composition methods do, so can sometimes be more efficient anyway [78]. Several authors $[58,106]$ have considered different symplectic Runge-Kuttas, but these have not been tested for accuracy and speed of convergence yet. If one is willing to form and invert Jacobians, Runge-Kuttas with real roots should be superior.

- Find the best symplectic Runge-Kuttas at each order.

For solving the implicit equations, the authors have always been content to extrapolate $x^{n+1}$ from previous time steps, and then solve $x^{n+1}=x^{n}+$ $f\left(x^{n}, x^{n+1}\right)$, say, by iteration to roundoff error-probably unbeatable as $\Delta t \rightarrow 0$, but high-order methods were not made for this. In tests, one should consider large, strongly-coupled systems such as those of molecular dynamics and discretizations of PDE's (see $\S 11$ ). In any event, iterating to roundoff is wasteful; one would prefer to only solve the equations to within the local truncation error.

- Develop iterations for the standard implicit symplectic methods which are symplectic and preserve equivariances of the original method after any number of iterations.

\section{Generating functions}

On a linear symplectic space there are many different kinds of generating functions. In addition to the four traditional types, Feng $[31,32]$ has provided a general discussion and described several non-conventional examples. These different types of generating functions may have different applications. For example, to generate the identity map from a generating function of the first (" $q, Q$ ") kind requires a singular generating function [18]. 
- What are the advantages of the specific types of generating functions? In particular, what are the advantages or disadvantages of using a singular generating function?

Ge [38] has shown that generating functions of the first kind respect symmetries well for cotangent bundles of nonlinear configuration spaces. Namely, they can be used to generate integrators which preserves the momentum corresponding to all cotangent lift symmetries, not just linear ones as for generating functions of the third (" $q, P$ ") kind. This leads to the Ge-Marsden [42] generating function for Lie-Poisson systems. Indeed, Ge [39] has generalized this construction to show that there is a generating function theory and a HamiltonJacobi equation, which in theory can be used to construct symplectic integrators for arbitrary Poisson manifolds. This construction uses the symplectic groupoid which exists locally over every Poisson manifold [21!; the attendant structures are just those that give rise to a generating function for the Poisson map.

For regular quadratic Lie algebras (those with a nondegenerate $A d$-invariant quadratic form), there is a generating function whici generates the identity transformation on the dual of the Lie algebra. However, it uses the coadjoint action, not of the identity group element but a family of group elements such that each $g(\mu)$ is from the coadjoint isotropy group of $\mu$. Namely, $A d_{g(\mu)}^{*} \mu=\mu$. This seems a bit unsatisfactory, although numerical experiments shows that the method works well [19].

- Is there a generating function for Lie-Poisson maps which generates the identity map via the coadjoint action of the identity group element?

A possible solution lies in the more general construction of Lagrangian submanifolds by Morse families [69]. Bear in mind that such a technique will not be of much use if it entails a substantial increase in computational complexity, as generating function methods already have inadequate efficiency [8].

For matrix Lie groups, the group can be thought of as a submanifold of a linear space which is acted on linearly by the group, and the Andersen constraint algorithm has a generating function of the third kind [80]. Indeed, there is a generating function that easily generates the identity map by the coadjoint action of the identity group element in this case. See $\$ 10$ for further discussion of symplectic constraint algorithms.

\section{Multistep methods}

The failure of symplectic methods to use information from past time steps leads to their needing more function evaluations than nonsymplectic methods, particular at orders more than four. Unfortunately, the most useful definition of symplecticity for multistep methods has not yet been arrived at. Some experimentation with actual systems is called for.

Start with a Hamiltonian system on a symplectic manifold $M$. An $n$-step method on $M$ may be written as a one-step method on $M^{n}$. The continuous system can also be extended to one on $M^{n}$ with the product symplectic structure. Ge and Feng [37] have investigated the circumstances under which one can preserve the product symplectic structure on $M^{n}$. Eriola and Sanz-Serna [29] and Ge [40] have investigated more general symplectic structures on $M^{n}$, 
and Bochev and Scovel [10] have shown that the symplecticity of these methods follows from their preservation of quadratic invariants.

But not every such system on $M^{n}$ arises from one on $M$, so preserving the product symplectic structure cannot be expected to lead to good results. For example, the two-step method $x_{i+1}=x_{i-1}+2 t X\left(x_{i}\right)$ preserves the product symplectic structure on $M^{2}[32,29]$, but has none of the nice properties one associates with symplecticity. Applied to integrable $m$-degree-of-freedom systems, the method does preserve invariant tori, but they have dimension $2 \mathrm{~m}$ in the $4 \mathrm{~m}$ dimensional space $M^{2}$, and do not project to subsets of $M$ close to the original tori, even as the time step tends to zero. What is needed are some extra integrals in the new directions, combined with an understanding of the relevant subspaces and corresponding projections, so that the invariant tori have dimension $m$ and do project nicely.

Feng [125] has proposed the completely different criterion that the induced one-step method (the map $m: M \rightarrow M$ satisfying the multistep method) should be symplectic in the usual sense. This sounds extremely appealing, but unfortunately Tang [125] has shown that no linear multistep methods can satisfy such a stringent criterion. Of course some general linear methods satisfy this criterion, namely, those derived from the composition of symplectic one-step methods; are there any others?

- Which multistep methods satisfy Feng's criterion?

However, one should also take into account that the initialization of the data to $M^{n-1}$ from a single initial value in $M$ might be determined by a different map than $m$. So multistep methods are functionally dependent on the initialization technique. Consider a two-step method for simplicity, in which $x_{3}$ is given as a function of $x_{1}$ and $x_{2}$. If the initial conditions are selected by $x_{2}=m\left(x_{1}\right)$, the sequence of points is that determined by the symplectic map $m$. However, if the initial conditions are selected by another mapping $x_{2}=f\left(x_{1}\right)$, this is not the case. We took the composition of two midpoint rules in the form

$$
x_{3}=x_{1}+t\left(X\left(\frac{x_{1}+x_{2}}{2}\right)+X\left(\frac{x_{2}+x_{3}}{2}\right)\right),
$$

which is symplectic in Feng's sense: its equivalent one-step method is the midpoint rule. Applied to the pendulum, we observed that as $f$ is perturbed from the midpoint rule, each invariant curve bifurcates into two curves, with successive iterates alternating between the two. This suggests that the map corresponding to two time steps might be symplectic.

MacKay [71] has proposed the criterion that if $\xi^{i}$ and $\eta^{i}$ are vectors such that $\omega\left(\xi^{1}, \eta^{1}\right)=\ldots=\omega\left(\xi^{n}, \eta^{n}\right)=c$, where $\omega$ is the symplectic two-form, then one should have $\omega\left(\xi^{n+1}, \eta^{n+1}\right)=c$ also.

The whole question remains one of the most important in symplectic integration.

- Find a criterion for symplecticity of multistep methods, and methods satisfying it, that leads to efficient methods with nice properties for Hamiltonian systems. 


\section{Preservation of integrals}

To preserve integrals one generally appeals to the theorem of $\mathrm{Ge}$ [38] that to do so up to a constant it is necessary and sufficient for the algorithm to be equivariant with respect to the symmetries generating the integrals. But there may be other approaches. Suris [121] has shown how making the Hamiltonian depend parametrically on the time step can lead to completely integrable versions of the standard map (i.e., $x^{j+1}-2 x^{j}+x^{j-1}=t^{2} f\left(x^{j}, t\right)$ ). Moser and Veselov [87] showed that a standard Lagrangian discretization of the free rigid body on $T^{*} S O(n)$ is not only completely integrable but has all the same integrals as the continuous system. Curiously, viewing the free rigid body as a free particle constrained to lie in $T^{*} S O(n) \subset T^{*} \mathbb{R}^{n \times n}$ and applying the symplectic constraint. algorithm of Andersen [3] gives the same map [80]. We do not know if this is more than a coincidence. Because the integrals do not depend on the time step, they are still preserved by higher-order maps formed by composition.

There are also the integrable discrete versions of integrable evolution equations such as the nonlinear Schrödinger equation and the Korteweg-de Vries equation. Some have integrable time discretizations too, although these are not usually local. [2]. Such equations are derived by discretizing the scattering problem of the continuous system, an approach which could be investigated from the point of view of symplectic integration. They usually preserve not the original symplectic structure, but a nearby one-an understandable price to pay for conserving energy exactly. The $Q R$ eigenvalue method can be viewed as a symplectic integrator conserving all integrals of the Toda lattice in Lie-Poisson form [124]. This, and the Moser-Veselov matrix factorization techniques [87], should be understood from the point of view of symplectic integration.

If a system were exceptionally sensitive to errors in a known integral, it might be worth enforcing it implicitly. One would add the integral as a constraint, satisfied by a Lagrange multiplier, which would naturally be zero for the timecontinuous problem. One needs an integrator which leaves invariant all the level sets of the integral, and is still symplectic in the usual sense-a slightly different criterion than that used in integration with constraints $(\$ 10)$.

- In which ways can integrals be conserved? Is it worth weakening the symplectic condition by requiring only preservation of a perturbed symplectic structure, if it enables conservation of more integrals?

\section{Variable time steps}

Standard methods can sometimes beat symplectic methods for pointwise accuracy, even at low order, if the system contains widely varying time scales in different parts of phase space. The reason is that the standard methods can adaptively change the time step. If one tries to, say, halve the time-step in a symplectic integration when $\left\|H^{\prime \prime}\right\|$ exceeds a certain threshold, the resulting map is not even continuous at the threshold hypersurface, and hence not symplectic. KAM tori are broken and good long-time behavior is lost [13].

Just as the time-t map of a canonical Hamiltonian system is symplectic, so its time-t $(q, p)$ is symplectic with respect to a non-canonical symplectic form. This suggests that this latter form should be preserved by a numerical method, and 
that $t(q, p)$ should be depend locally and smoothly on $q$ and $p$. This approach has not been developed to our knowledge. There is a theory of time-dependent transformations in Hamiltonian systems, explored by C'arineña and coworkers [15]. which may be useful. Another, similar, approach would be to integrate $J d H /\|J d H\|$, which also preserves a non-canonical symplectic form. It has been suggested that the ultimate criterion is that the numerical method should be the time- $t(q, p)$ flow of a Hamiltonian system close to the original one. For this and further discussions, see $[110,117]$.

In the integration of Hamiltonian systems with a nonstandard symplectic structure, such as the Ablowitz and Herbst [1] study of the Schrödinger equation, Darboux charts are useful to bring the system to the standard canonical form. Our preliminary investigations show that often the mapping to symplectic variables is explicit but its inverse is not and it is the inverse that is required to produce the Hamiltonian in the new variables. This presents algorithmic difficulties that require further investigation.

To date the only successful approach is that of Skeel and Biesiadecti [118] and MacEvoy and Scovel [70]. They partition the Hamiltonian so that it is a sum of fast and slow terms, the fast terms being zero outside some region of phase space. The slow terms are integrated with a large time step unless that step puts that orbit in the fast region, in which case it must be retaken with a smaller time step. MacEvoy and Scovel [70] join the two regions smoothly by a partition of unity. To date these algorithms have not been developed to the point where one can make a fair comparison with standard variable time step schemes. Simó [115] has suggested taking the Kepler problem (a particle in $\mathbb{R}^{2}$ under a central inverse-square force) with eccentricity 0.999999 as a good test of a variable time step method.

- Develop variable time step symplectic integrators so that they are competitive for pointwise accuracy with standard methods for the Kepler problem, while retaining the good long-time behavior of constant time step symplectic methods.

\section{Constrained systems}

\subsection{Dirac constraints}

Suppose that we are given a Hamiltonian on a symplectic manifold $M$ and a submanifold $N \subset M$ to which we would like to constrain the dynamics. Suppose that by the method of Dirac constraints we have a found a set of constraint functions $\phi: M \rightarrow V$ (where $V$ is a linear space) such that $N=\{x \in M$ : $\phi(x)=0\}$ and the matrix $\left\{\phi_{i}, \phi_{j}\right\}$ is nonsingular. The problem is to construct a map from $N$ to $N$ approximating the constrained dynamics and preserving the indiced symplectic structure on $N$. Frequently the system in invariant under a symmetry group, in which case the integrator should preserve the corresponding momentum.

This has been addressed and solved only in the case of holonomic constraints $[80,66,98,99,60]$, and when the symmetry group acts by cotangent lifts of linear transformations. That is, when $M=T^{*} Q$ is a cotangent bundle and the constraints are divided into two sets, the primary constraints $\phi_{1}(q)=0, q \in Q$, and the secondary constraints $\phi_{2}(q, p)=d \phi(q) \partial_{p} H(q, p)=0$. In the Lagrangian picture, holonomic constraints restrict the motion to $T^{\prime} N \subset T Q$ [75]. 
- Develop momentum-preserving constrained symplectic integrators for general constraints and for general symmetry groups.

Also to be understood is the situation with regard to symplectic integration when one is given an arbitrary singular Lagrangian on an arbitrary manifold.

At present, the general theorems concerning momentum preservation require the constraint functions to be group invariant. However, in the case of the Lagrange top, McLachlan and Scovel [80] found only degenerate invariant constraint functions. Since nondegeneracy is essential for the constraint algorithm, they dropped one of the invariances; but it turned out that the integrator preserved its momentum anyway.

- What special properties are required of the constraint function in order that a momentum-preserving symplectic integrator may be determined?

\subsection{Fewer implicit steps}

The currently recommended algorithms for holonomically constrained separable Hamiltonian systems $[3,66,60,98,80]$ involve solving a set of implicit equations to enforce the primary constraints each time $q$ is updated. For a higher-order method formed by composition, this means many implicit steps. (The secondary constraints can be enforced with an explicit step.) Is it possible to do better by only enforcing the constraints at the end of each complete time-step? Although only a small $\mathcal{O}\left(t^{p+1}\right)$ projection to the constraint surface is needed (here $t$ is the time step and $p$ the order of the method) the crucial point is to make the entire time-step map symplectic on the constraint surface.

- Are there constrained symplectic integrators with fewer implicit steps than those currently in use?

\subsection{Generating functions}

The proof of McLachlan and Scovel [80] that the constraint algorithm of Andersen is symplectic relied on a generating function representation. This representation can be manipulated to generate higher order-methods.

In addition, the Dirac formula can be used to determine the exact Lagrange multipliers for the continuous system, at the cost of more derivatives and matrix inversions. In return the number of iterations iterations on the implicit step is reduced. At present we are using Lagrange multipliers extrapolated from previous time steps, combined with secant or Newton-secant iteration to roundoff error.

- Can one modify the Hamiltonian or predict the value of the Lagrange multipliers to make the implicit step more efficient? Are there methods which are symplectic after a finite number of iterations on the multipliers?

Indeed, the generating function picture is far from complete.

- Understand in depth the symplectic geometry of the generating function method for holonomic constraints.

It is hoped that a full understanding of this problem should lead to a method for nonholonomic constraints. 


\subsection{Automatic prescrvation of quadratic constraints}

In many physical systems, such as rigid body and molecular dynamics, the constraints are quadratic functions, being based on preservation of Euclidean lengths. Because symplectic Runge-Kutta methods, such as the midpoint rule, preserve quadratic integrals $[20,109,23]$, these seem to be good candidates to integrate such quadratically-constrained systems. Specifically, the constraints on the configuration variables are quadratic and the secondary constraints $\phi(q, p)$ have quadratic extensions to the full space. However, the application is not direct because only the zero level set of the constraint function $\phi(q, p)$ is invariant. Such an "integral" would not be preserved.

- For which quadratically-constrained systems can the Hamiltonian and the constraint functions be extended to a neighborhood of the constraint surface such that the $c$ nstraints are true quadratic integrals of the extended Hamiltonian system?

For the rigid body and the double spherical pendulum, for example, to leave all level sets of the natural constraint functions invariant requires a rightinvariance of the extended Hamiltonian which we have not been able to obtain $[80]$.

Another possible application involves Lie-Poisson systems. Many Lie groups can be written as submanifolds of linear spaces defined by quadratic constraints. For example, $S O(n)=\left\{q \in \mathbb{R}^{n \times n}: q^{t} q=1\right\}$. In this case symplectic Runge-Kutta methods can be used to approximate the exponential map and compute group elements. For so $(n)$, the midpoint rule generates the Cayley transform between $\mathfrak{s o}(n)$ and $S O(n)$ : for $A \in \mathfrak{s o}(n),(I-A)^{-1}(I+A) \in S O(n)$. It is tempting to use this fact in conjunction with the coadjoint action to construct Lie-Poisson integrators for matrix Lie groups determined by quadratic constraints. For example, consider the integrator $z \mapsto Z$ where

$$
Z=A d_{R(d H((z+Z) / 2))}^{*},
$$

and $R(x)$ is the Runge-Kutta integrator applied to the Lie algebra element $x$. The range of $R$ is in the group, so that it is natural to ask if this integrator is Lie-Poisson. Our preliminary calculations indicate that it is not, but that does not mean that there is not some alternative Lie-Poisson form. There are many other integrators based on this idea. Probably it would be good to test them numerically first.

- Use quadratic preserving Runge-Kutta methods to generate Lie-Poisson integrators.

\section{Approximation of infinite-dimensional Poisson manifolds}

For Hamiltonian field theories, the phase space is an infinite-dimensional Poisson manifold [128]. For an introduction to this subject, see Olver [91]; for applications to fluid mechanics, see Salmon [108] and Arnol'd and Khesin [7]. To numerically simulate these equations a finite dimensional truncation is necessary.

- Should a finite dimensional truncation of an infinite dimensional Hamiltonian system be Haniltonian? 
Specifically, we want to be able to let $\Delta t=f(\Delta x)$ ( $f$ being determined by stability criteria) and study

$$
\text { " } \lim _{\Delta x \rightarrow 0} \lim _{t \rightarrow \infty} "
$$

Usually in numerical analysis these two limits are reversed, or $t$ is held fixed. But most real computations use (3), because global errors can be kept small only for impractically short times. It is implicitly hoped that qualitative and statistical behavior of the PDE is mimicked by the finite-dimensional model, Hamiltonian or not. At this early stage, some numerical experimentation is in order.

In the hope that there are good positive answers the the above question, we now discuss the approximation of Poisson manifolds. After the truncation of the phase space has been determined, there is still the question of choosing the best Hamiltonian.

If the Poisson bracket is constant, i.e., independent of the field variables, truncation is trivial, because any antisymmetric matrix is a Poisson tensor. As in finite dimensions, this is the most common case. Thus we immediately get Hamiltonian truncations for nonlinear wave equations, the nonlinear Schrödinger equation, the Davey-Stewartson and Boussinesq equations, and so on. In fact, with periodic boundary conditions, all of these have a two-term splitting, so the design of the Hamiltonian method is finished. Some questions of numerical analysis are considered in [77]. If the resulting Courant-type stability restriction (roughly two time-steps per period of the fastest wave) are too severe, the fast modes should be removed in the Hamiltonian, not in the integrator.

For equations of the form $\dot{u}=\partial_{x}(\mathcal{L} u+f(u))$, where $\mathcal{L}$ is a linear operator, it is annoying that being able to solve $\dot{u}=\partial_{x} f(u)$ by characteristics is no help: we must solve the discretized equation $\dot{\mathbf{u}}=D \mathbf{f}(\mathbf{u})$. If $D$ has bandwidth $b$ above the diagonal, then we can do a $b+1$-piece splitting (e.g., odd-even splitting for $b=1$ ), and $b /(b+1)$ of the unknowns evolve under a given piece. During a (first-order) time step, each unknown is stepped $b$ times, which in unappealing-particularly if $D$ is full, as for spectral methods. We have the paradoxical situation that an implicit method using FFT's would be asymptotically faster than an explicit method.

The simplest truncation for Hamiltonian systems on $T^{*} Q$, where $Q$ is the configuration space, is to determine a Hamiltonian system on $T^{*} N$ where $N$ is a finite-dimensional submanifold of $Q$. This is done in the Lagrangian description by restriction to the tangent space $T N \subset T Q$. Then the Legendre transform maps to a Hamiltonian system on $T^{*} N$. This is the technique proposed by Holm et al. [50]. However, it can destroy symmetries of the system such as the particle relabeling symmetries of fluid mechanics.

Many other evolution equations of physics-Eulerian inviscid fluids, the Vlasov-Poisson equation, the Korteweg-de Vries equation-can be written as LiePoisson systems. Their phase space is the dual of some infinite-dimensional Lie algebra. To spatially truncate such systems to a set of Lie-Poisson ODE's leads naturally to the question of finding finite-dimensional approximations of these Lie algebras. The approximation should converge to the infinite-dimensional algebra in some useful sense (perhaps its structure coefficients converge pointwise) and inherit as much structure as possible: all the discrete Casimirs (such as helicity in the $3 D$ Euler equations), and an increasing number of the continuous ones (such as the generalized enstrophies in the 2D Euler equations) as the approximation improves. It is hoped that in this way statistical properties of 
the discrete system will approximate those of the continuous system, although this is little more than a hunch at present $[59,133,9]$. For example, how does symplectic integration of Zeitlin's truncation of the Euler equation converge to Joyce-Montgomery [57] states? Is there a Joyce-Montgomery equation for negative temperature states on $\mathfrak{s u}(n)$ ? What is the effect of different Hamiltonians, such as the one presented in Dowker and Wolski [25]? How important is it to preserve the higher Casimirs in the numerical simulation?

Truncation of Poisson manifolds is a very difficult and fundamental problem, and little progress has been made. Certainly there is no general theory. Simple finite differences and spectral methods do not work, even elegant ones such as Arakawa's energy- and enstrophy-preserving schemes [4, 5]. We can only summarize the presently known approaches.

\subsection{The sine bracket}

For the algebra of Hamiltonian vector fields on the torus $\mathbb{T}^{2}$, the Lie bracket in Fourier space takes the form

$$
\left[\omega_{\mathbf{m}}, \omega_{\mathbf{n}}\right]=(\mathbf{m} \times \mathbf{n}) \omega_{\mathbf{m}+\mathbf{n}}
$$

where $\mathbf{m}=\left(m_{1}, m_{2}\right)$ is a wavenumber and $\mathbf{m} \times \mathbf{n}=m_{1} n_{2}-m_{2} n_{1}$. The sinebracket truncation retains the modes with $-M \leq m_{1}, m_{2} \leq M$ and perturbs the bracket to

$$
\left[\omega_{\mathbf{m}}, \omega_{\mathbf{n}}\right]_{\sin }=\varepsilon^{-1} \sin (\varepsilon \mathbf{m} \times \mathbf{n}) \omega_{\bmod (\mathbf{m}+\mathbf{n})}
$$

where $N=2 M+1, \varepsilon=2 \pi / N$, and mod $(m)$ reduces $m$ modulo $N$ to the lattice $M \leq m_{1}, m_{2} \leq M[30,51,52]$. Notice how the sine bracket converges to the continuous bracket as $N \rightarrow \infty$ for fixed $\mathbf{m}, \mathbf{n}$. (5) turns out to define the Lie bracket of $\operatorname{su}(N)$, so this algebra has $N-1$ Casimirs, the $n$th one of which tends to the $n$th Casimir of (4) as $N \rightarrow \infty$ for fixed $n$. Clearly the precise sense in which $S U(N)$ approximates the group of symplectic maps of $\mathbb{T}^{2}$ remains to be established!

This algebra provides a Lie-Poisson truncation of, among other systems, the 2D Euler equations with periodic boundary conditions $[9,25,82,133]$. It seems to be unique, and is related to the Moyal bracket of quantum mechanics [34]. It allows truncations of the shallow water equations in Eulerian [43] and Lagrangian [103] formulations, although these have not been tested. Perhaps one should first study the sine-bracket independently of its application to fluid mechanics, for instance, by choosing Hamiltonians which give smoother flows.

- Is the sine bracket useful in numerical methods?

\subsection{Moment truncations}

For Vlasov-Poisson-type systems whose phase space consists of densities $f$ on a base symplectic manifold $P$, one can define the moments $f^{\alpha}=\int_{P} x^{\alpha} f d x$ and the induced Poisson bracket $\left\{f^{\alpha}, f^{\beta}\right\}=f^{\gamma}$, where $\gamma=\left\{x^{\alpha}, x^{\beta}\right\}_{P}$. (Here $\alpha$ is a multiindex; the geometric point of view is discussed below.) There are two trivial truncations of this infinite-dimensional Poisson space which may be useful. Notice that $|\gamma|=\max (0,|\alpha|+|\beta|-2)$ so that the algebra has two subalgebras, one consisting of moments of order $0 ; 1$, and 2 , and one of moments of orders 2 
and higher. In the first case no truncation is required, and Rouhi (in [104]) has used this to approximate elliptical vortex patches; one can also get the "next correction" to point vortices in this way. The second case has an elementary finite-dimensional truncation to moments of order 2 through $n$ [19], discussed below. Such a truncation is useful for localized distributions such as arise in accelerator dynamics [16]. But if one wishes to retain moments of order 0 through $n$, perhaps with multiple base points, a more sophisticated approach lcading to mixed Eulerian-Lagrangian schemes, outlined $: \downarrow \$ 11.4$, is needed.

More applications of these truncations should be explored, if only because truncations are rare.

- Apply moment truncations to fluid mechanics and to general IIamiltonian PDE's.

\subsection{Other approaches}

Staying in the class of Lie-Poisson systems, the above exhausts the curront ideas, and more are desperately needed. The sine bracket exists only for the algebra of Hamiltonian vector fields on the sphere, the $2 n$-torus, and possibly on other symplectic coadjoint orbits [51].

If one changes variables by the discrete Fourier transform, the sine bracket transforms to a bracket on functions on a lattice. However, this bracket is nonlocal.

- Is there a local Lie bracket on functions on a discrete representation of the plane approximating the usual Poisson bracket?

It is possible that there is none, due to the rigidity of Lie algebras. One cannot get rid of this problem by going to a nonlinear Poisson bracket since the Poisson structure transverse to the symplectic leaves is linearizable to a LiePoisson structure [128], so there must be Lie algebras present. However, if we could find a local Lie bracket, the next question would be how to deal with boundary conditions. For example, to do simulations of the Euler equations on the sphere it would be nice to have a Lie algebra which approximates the Poisson algebra of Hamiltonian functions which vanish on continents. This would also be useful for the boundary conditions imposed by the walls in an accelerator.

There is also the work of Dubrovin [27] on discrete Poisson brackets. For a certain bracket on the field variable $u: \mathbb{R} \rightarrow \mathfrak{g}$, where $\mathfrak{g}$ is a Lie algebra, he was able to replace the domain $\mathbb{R}$ by a discrete lattice only by deforming the range $\mathfrak{g}$ to a Poisson-Lie group. Workers in quantum groups have pointed out the similarity between the processes of quantization and discretization [94].

There is no known approximation for the algebra of divergence-free vector fields on $\mathbb{T}^{3}$, for example, which would be needed to study the 3D Euler equations. To make the formulas easier one might study the algebra of vector fields on the circle, for which the Poisson operator is $u \partial_{\theta}+\partial_{\theta} u[78,94]$.

However, one can leave the category of strict Poisson truncation. For the algebra of vector fields $u(\theta)$ on the circle, the change of variables $u=v^{1 / 2}$ makes the Poisson operator constant [77]. Similar changes of variables exist in other cases [84] but due to their hodograph nature may not be of practical use. One could search for canonical variables on particular symplectic leaves, or find 
symplectic (Clebsch) variables on the whole space $[74,132]$. This latter approach is unappealing as the truncation will generally destroy the gauge symmetry of the symplectic variables, leading to an excessively large phase space. In any event, there is no Darboux theorem for PDE's yet [90]. Lagrangian formulations of fluids can be canonical, so one should explore as many such formulations as one can find. The "velicity" method $[92,12]$ is interesting in this regard. See also Virasoro [126]. Lagrangian vorticity and velocity-vorticity formulations should be studied. The three-dimensional vortex methods currently in use do not appear to be canonical [129]; extending them to be so would be an important development. Symplecticity is of importance in stellar dynamics too [83], so Hamiltonian truncations of its Vlasov-Poisson formulation should be useful.

The method of Dirac constraints has proved useful in deriving simplified evolution equations [107]; is it possible to get a sensible Hamiltonian truncation by constraining the fluid motion to a finite-dimensional submanifold?

\subsection{Augmentation and decoupling of semi-direct products}

Although there is no approximation of the group $D$ of volume-preserving diffeomorphisms at present, there is a useful approximation of the dual of its Lie algebra as a Poisson manifold. Following Scovel and Weinstein [113] we augment to a slightly larger space and use an isomorphism to direct products of Poisson manifolds, each which have Hamiltonian truncations.

Consider the subgroup $D_{0}$ consisting of those diffeomorphisms of the 3-torus $\mathbb{T}^{3}$ which preserve the origin. Let $N_{k}$ denote the normal subgroup of $D_{0}$ whose elements $d$ are such that the lst through $k$ th derivatives of $d(x)-x$ vanish at the origin. Then, $D_{k}=D_{0} / N_{k}$ can be thought of as an approximation to $D_{0}$. It is the finite-dimensional group consisting of the $k$ th-order jets of volume-preserving diffeomorphisms which preserve the origin. Likewise, the dual of the Lie algebra $D_{k}$ is a space of vector moments up to $k$ th order.

However, what we wish to approximate is $\mathcal{D}^{*}$. We do this by pulling back the Hamiltonian to the semidirect Poisson manifold $\mathcal{D}^{*}\left(S \mathbb{T}^{3}\right.$ by the Poisson map consisting of projection onto the first factor. Then pull back by a Poisson isomorphism $T^{*} \mathbb{T}^{3} \oplus \mathcal{D}_{0}^{-} \rightarrow \mathcal{D}^{*}\left(S \mathbb{T}^{3}\right.$ which decouples the semidirect product Poisson manifold into the direct product of Poisson manifolds. This determines a Hamiltonian system on $T^{*} \mathbb{T}^{3} \oplus \mathcal{D}_{0}^{*}$ for which the first factor is finite dimensional and the second is truncatable. The new phase space has the physical interpretation of the replacement of the momentum density by a single particle plus the 0th through $n$ moments of the distribution about that particle [113]. The determination of a Hamiltonian on the truncated space would follow by pulling back by the Poisson map $T^{*} \mathbb{T}^{3} \oplus \mathcal{D}_{k}^{*} \rightarrow T^{*} \mathbb{T}^{3} \oplus \mathcal{D}_{0}^{*}$ which is the adjoint to the one induced by the projection $\mathcal{D}_{0} \rightarrow \mathcal{D}_{k}$. However, the Hamiltonian is not usually well defined on this image since an image point consists of sums of delta functions and their derivatives, which we call a multipole. Consequently, we pull back by another map $\phi: T^{*} \mathbb{T}^{3} \oplus \mathcal{D}_{k}^{*} \rightarrow T^{*} \mathbb{T}^{3} \oplus \mathcal{D}_{0}^{*}$ such that when it is composed with a natural projection $T^{*} \mathbb{T}^{3} \oplus \mathcal{D}_{0}^{*} \rightarrow T^{*} \mathbb{T}^{3} \oplus \mathcal{D}_{k}^{*}$ should give the identity. Probably $\phi$ should the identity in the first factor.

- What are the optimal $\phi$ 's for a specific physical system?

This technique appears to work well when the distribution in $\mathcal{D}^{*}$ is concentrated about a point and can be well-represented by its first few moments. One 
can incorporate many of these nultipoles by first pulling back to the direct sum $\sum \mathcal{D}^{*}$ by the map $\sum \mathcal{D}^{*}-\mathcal{D}^{*}$ that simply sums up the distributions. Since the Lie-Poisson structure on $\mathcal{D}^{*}$ is linear, this summation is a Poisson map. Consequently, we have a progression of Hamiltonian truncations, from one multipole with a large number of moments to a large number of multipoles with a small number of moments.

- For a specific problem, what is the optimal balance between the number of multipoles and the number of moments retained for each?

In the truncation of the shallow water equation, Ge and Scovel [43] use a Poisson isomorphism

$$
T^{*} F^{*} \oplus \mathcal{D}_{\mu}^{*} \rightarrow \mathcal{D}^{*}\left(S F^{*}\right.
$$

where $F^{*}$ is the space of positive mass densities, $\mathcal{D}^{*}$ is the space of momentum densities, and $\mathcal{D}_{\mu}^{*}$ the space of vorticities. The direct product space can be truncated by truncating $F^{*}$ by a finite element. method and approximating $\mathcal{D}_{\mu}^{*}$ by $\mathfrak{s u}(n)$ for $n$ large [30]. The difficulty here is that the isomorphism involves a map determined to exist by a theorem of Moser [88] on volume elements. Liao et al. [68] have been developing a numerical approximation of this map based on a deformation technique, and Cullen et al. [22] have successfully used the Monge-Ampere equation. The latter method appears to be more amenable to differentiation.

- Construct a numerical approximation to a map corresponding to Moser's [88] theorem on volume elements that makes the Ge-Scovel [43] shallow water approximation implementable.

\section{Theoretical performance}

\subsection{The KAM theorem}

Consider a smooth autonomous Hamiltonian of the form

$$
H_{0}+\varepsilon H_{1}+(\Delta t)^{p} H_{2}
$$

where $H_{0}$ is integrable, $H_{0}+\varepsilon H_{1}$ is not, and $H_{2}$ is the first term in the perturbation due to a symplectic integrator. If $\varepsilon=0$, KAM theory implies that most of the invariant tori of the system $H_{0}$ are preserved by the integrator, and that the fraction preserved tends to 1 as $\Delta t-0$. What can we do when $\varepsilon \neq 0$ ? A direct application of KAM theory only shows that most of the tori of $H_{0}+\varepsilon H_{1}$ are still preserved when $\Delta t \neq 0$, by comparing back to $H_{0}$. It seems that one should be able to get more; see for example the informal discussion in [78].

- Rigorously investigate Hamiltonians of the form (12.1) as $\Delta t-0$ for $\varepsilon$ fixed but arbitrarily small. 


\subsection{Extensions of Hamiltonian stability theorems}

MacKay [ $\vec{i}]$ ] has pointed out that the reason symplectic integrators preserve energy well over long simulations follows from the results of Arnol'd [6] and Neishtadt [89]. However, this result on the adiabatic invariance of the energy appears to have some validity outside the realm of Hamiltonian mechanics. We also suspect that the KAM and Nekhoroshev theorems have counterparts in larger categories than Hamiltonian systems. Let us be more precise.

Some proposed integrators are not actually symplectic but appear to have the same stability characteristics as bona fide symplectic methods. For example, consider the integrators of Channell and Scovel [18] based on generating functions determined from the Hamilton-Jacobi theory. In truth, they do not determine symplectic maps. This can be seen as follows. Pick an initial point $z_{0}$. Then, determine the generating function about $z_{0}$ to generate the symplectic map from $z_{0}$ to the next point. Now consider another point $z_{1}$ near $z_{0}$. Its generating function is determined by a Taylor series about $z_{1}$, not $z_{0}$, so the generating functions do not match in general. Therefore, $z_{1}$ is advanced by a different symplectic map than $z_{0}$. Consequently, the method does not necessarily determine even a local symplectic map. However, their numerical experiments show that this method has the typical superior performance of symplectic integrators.

The domain where this integrator exists is probably something like the action groupoid $G \times Z$ where $G$ is the group of symplectic maps and $Z$ is the phase space, instead of the symplectic group relevant for the usual symplectic integrator. Actually, $G$ is more likely to be the local symplectic mappings, but we prefer not to confuse the issue any more than necessary here. For a review of groupoids and algebroids see Mackenzie [72]. The partially-defined multiplication in $G \times Z$ is $\left(g_{2}, z_{2}\right)\left(g_{1}, z_{1}\right)=\left(g_{2} g_{1}, z_{1}\right)$ if $z_{2}=g_{1}\left(z_{1}\right)$. This is natural, since if one starts at $z_{1}$ and determines a flow map $g_{1}$, then on the next step one begins at $z_{2}=g_{1}\left(z_{1}\right)$. This structure seems to be relevant for numerical analysis in general, not just for Hamiltonian mechanics. The analogue of the Lie algebra of Hamiltonians $\mathfrak{g}$ of $G$ for the groupoid is the Lie algebroid $\mathfrak{g} \times Z$ which is a vector bundle with fibres $g$ and base $Z$. It is not a Lie algebra, but there is a lie bracket on sections of this vector bundle. Now consider a Hamiltonian $H \in \mathfrak{g}$ and the constant section $Z \rightarrow \mathfrak{a} \times Z: z \mapsto(H, z)$.

- If the KAM or Nekhoroshev theorems are applicable to the Hamiltonian system determined by $H$, are there analogues of these stability theorems for sections of the action algebroid $g \times Z$ which are small perturbations of the constant section $z \mapsto(H, z)$ ? Is there an adiabatic invariance theorem for such an extension?

As mentioned above, there is numerical evidence to support the existence of these theorems. In fact, the beginnings of a KAM extension can be outlined: since the evolution of $H$ is by quasi-periodic motion, substitution of this motion into the z dependency of the perturbation gives a quasi-periodic time-dependent perturbation of $H$ to use as a base motion.

There appear to be other areas where such extensions might exist. For example, in the work of MacEvoy and Scovel [70] concerning the development of variable time step methods, normalizing the Hamiltonian to be zero at the initial conditions has some stabilizing effect, but this cannot be done globally in the 
phase space. Probably the natural framework for proving stability theorems is the action algebroid.

\subsection{Reversible adiabatic invariance theorem}

It is known that there is a KAM type theorem for $R$-reversible systems. However, [70] and [77] it is observed that integrating an $R$-reversible Hamiltonian system by an $R$-reversible nonsymplectic integrator preserves the energy well over long simulations. See [102] for a review of the interactions between reversibile and Hamiltonian systems.

- Is there an adiabatic invariance theorem for reversible perturbations of reversible Hamiltonian systems?

When the original Hamiltonian system is integrable, one can ask whether all the integrals of motion are adiabatic invariants for $R$-reversible perturbations.

- Is there a Nekhoroshev theorem for reversible perturbations of reversible integrable Hamiltonian systems?

Reversibility extends naturally to multistep methods, and Saha and Trenıine (see, e.g., [105]) have developed and applied such methods usefully to the solar system. Presumably one only obtains good behavior near the symmetry plane. More generally we propose:

- Study $R$-reversible perturbations of $R$-reversible Hamiltonian systems.

\subsection{Chaos}

It is impossible to track orbits whose Lyapunov exponents are of order one for long times. Typically, the chaotic and quasiperiodic orbits are intermingled in Hamiltonian systems; indeed, there are no known completely hyperbolic polynomial Hamiltonians. So, at least the boundaries of the chaotic regions will be better preserved in a symplectic than in a nonsymplectic integrator. But what about the flow in the chaotic region itself?

Because individual orbits cannot be tracked accurately, the chaotic case should be symplectic integrators' forte. But so little is known analytically that it is hard to know what kind of result one should aim for. Instead, there is a result which is negative from our partisan point of view: for Anosov systems, all nearby flows are conjugate, not just the symplectic ones. Some numerical work might resolve the apparent contradiction.

- Measure statistical properties of chaotic orbits, and their dependence on symplectic and nonsymplectic perturbations.

\section{Dissipative systems}

- In the numerical simulation of slightly dissipative perturbations of Hamiltonian systems, does one simply give up the Hamiltonian structure? 
There is evidence to the contrary. Unpublished work of the second author with $\mathrm{D}$. Umbarger used the fact that the Duffing oscillator has a time-dependent coordinate transformation into a time-dependent Hamiltonian system. They compared a fourth-order symplectic integrator in this moving frame with a Bulirsch-Stoer variable time step method: the two appeared to be of comparable quality.

Lewis and Marsden [67] describe a Harniltonian-dissipative normal form decomposition of a vector field and discuss how the Hamiltonian part provides an organizing center of the system. Kirchgraber et al. [64] observe that in a simple case that if the integrator is symplectic when the dissipation is turned off, the numerical center manifold is stable under the dissipative perturbation. Mielke [81] describes the Haniltonian structure inherited on center manifolds and Sleeman [119] shows that time-periodic solutions of reaction-diffusion equations can form Hamiltonian systems in the single spatial variable. Since the center manifold is a component of the system with the exponential decay factored out we ask the following questions.

- When is the flow on the center manifold of a dissipative system Hamiltonian? When is the flow on an inertial manifold Hamiltonian?

Such a situation is surely not true in general, but it would be interesting to know if there are any important special cases.

Grmela [46] and Morrison [85, 86] provide bracket formulations for Hamiltonian plus gradient systems and Vujanovic and Jones [127] have described a variational form for nonconservative systems. What is missing is an invariant structure whose preservation provides superior numerical simulations.

For a Hamiltonian system on $M$, the graph of the vector field is a Lagrangian submanifold of $T M$ with respect to the tangent symplectic structure. This is the infinitesimal version of the fact that the graph of the flow map of a Hamiltonian system is Lagrangian in the symplectic manifold $M \times M_{-}$, where $M_{-}$is $M$ with the negative of its symplectic form. Cantrijn [14] has shown that the graph of a gradient perturbation of a Hamiltonian system in TM is Lagrangian with respect to a symplectic form on TM which is the tangent one altered by a term depending on the gradient perturbation. Unfortunately, this symplectic formulation does not seem to produce invariants at the map level, so it is unclear what its implications are for numerical simulations.

- Which dissipative systems have flow invariants whose preservation produces superior numerical simulations?

For any dynamical system, one can double the dimension of the space by the cotangent lift to obtain a Hamiltonian system. This does no good numerically as invariant tori are typically half the dimension of the symplectic manifold, so symplectic methods bring one back to the dimension of the original system. However, Nose [93] shows that molecular dynamics formulations with constant temperature may be presented in Hamiltonian form by the addition of two more variables.

- What is the structure of a dynamical system which allows a Hamiltonian formulation by the addition of a small number of additional variables? 


\section{Assorted issues}

The program of studying geometric structure in numerical methods has scarcely begun. We select just a few such structures for further comment.

\subsection{The discrete Lagrangian method}

Discretization of the Lagrangian description appears to be very old. Maeda [73] and Mackay [71] outline formalisms for discrete Lagrangian methods and describe the corresponding symplectic method. MacKay [71] points out that the method is symplectic with respect to a perturbed symplectic structure. Moser and Veselov [87] discretize the Lagrangian description of the free rigid body and determine a completely integrable approximation preserving the rigid body integrals. McLachlan and Scovel [80] show that application of a symplectic constraint algorithm to the free rigid body produces the Moser-Veselov integrator.

It is possible that in many cases either the Lagrangian formulation or the the Hamiltonian formulation might be superior with respect to numerical simulations.

- Investigate the quality of Lagrangian integrators, and their equivalent Hamiltonian forms.

\subsection{Reversible sysiems}

Many authors have pointed out how easy it is to construct reversible integrators $[77,78,98,111]$, and how important it is on general principles a priori. Most applications are in Hamiltonian systems. However, one should also

- Perform numerical experiments to investigate the importance of preserving reversibility with respect to one or multiple involutions.

This is important since one may not know the involutions explicitly, or even if they exist. Scovel [111] projects an arbitrary map onto $R$-reversible maps; this doubles the amount of work, so one would like to know whether it is worth it. See [102] for a review of reversibility and Hamiltonian systems.

\subsection{Volume preservation}

Volume-preserving flows arise in particle-tracking in incompressible fluids and in the standard spectral truncation of the 2D Euler equations. (Interestingly, this latter model also preserves energy and enstrophy - how well are they preserved by a volume-preserving scheme?) The space of divergence-free vector fields seems to be too large: no standard numerical methods, such as Runge-Kuttas, are volume-preserving for all such vector fields [95]. There is a generating function for volume-preserving maps [111], but it involves evaluating definite integrals of the vector field and does not preserve fixed points in general. In many systems such as the $A B C$ flows a splitting technique works well $[111,49,48,95]$.

- Develop efficient, general volume-preserving methods. 


\subsection{Symplectic lattice maps}

Often roundoff accumulation can become a problem even with the symplectic methods. Symplectic lattice maps have been studied $[97,63,62]$ and are a possible resolution of this problem. Earn and Tremaine [28] show that the elinination of roundoff error in this way can effect the statistical behavior of the simulation. Scovel [112] shows that often a symplectic map can be altered to a nearby symplectic map which preserves a lattice. It is obvious that the lattice maps should get more accurate as the lattice is refined from a crude lattice. However, as the lattice gets finer, the preservation of the lattice causes a high-frequency perturbation which may cause serious diffusion problems in the zero lattice spacing limit.

- For specific Hamiltonian systems, is there an optimal lattice spacing for a symplectic lattice map approximation?

\subsection{Standard methods}

For some dynamical systems, very high order Cowell methods are so accurate and so inexpensive that the local truncation errors can be made comparable to roundoff errors. Then (to within roundoff error) it is a symplectic multistep scheme in Feng's sense. See Hyman et al. [55] for a discussion of this matter.

- For a specific Hamiltonian system, and a fixed degree of accuracy of some property of the system, develop a criterion to determine when it is worth the extra affort to implement a symplectic integrator versus a traditional method.

\subsection{Shooting methods}

One-dimensional quantum mechanics problems often involve computing the eigenvalues of a Schrödinger operator. Leaving the eigenvalue as a parameter to be determined one can use a shooting method from the left-hand boundary to the right. The differential equations are Hamiltonian.

- What are the benefits of using a symplectic integrator in a shooting method?

\subsection{Elliptic equations}

Can one devise numerical approximations of the Div, Grad, and Curl operators that satisfy the vector identities Div $\circ \mathrm{Curl}=0, \mathrm{Curl} \circ \mathrm{Grad}=0$ exactly? The answer is yes and appears in Dodzuik [24] and Hyman and Scovel [54]. Triangulate the basic manifold by a chain complex. Then the coboundary operator $\delta$ is the discrete analogue of the exterior derivative $d$ and the discrete vector identities $\delta \circ \delta=0$ are consistent with the fact that the deRham map from the deRham complex to the cochain complex is a chain map. In a closely related strategy, Hyman et al. [56] develop and test finite volume approximations to differential operators on nonuniform grids based on Stokes's theorem.

If we impose a Riemannian structure, we can take adjoints and compute a Laplacian. In fact, Hyman and Scovel [54] show that the Hodge theorem still 
applies here in the sense that there is a discrete Hodge decomposition and the dimension of the kernel of the discrete version of the Laplacian on $k$-forms is the dimension of the $k$ th cohomology group of the manifold. However, at present these Laplacians are not local since the induced Hodge star operator is not local.

- Is there a local discrete Hodge star operator which has a local inverse?

- Are there chain map approximations to the exterior derivative that are more than first-order accurate?

- Can one quantify the benefits of using these techniques?

\section{References}

1. Ablowitz, M. J., and B. M. Herbst, On homoclinic structure and numerically induced chaos for the nonlinear Schrödinger equation, SIAM J. Appl. Math. 50 (1990), 339-351.

2. Ablowitz, M., and H. Segur. Solitons and the Inverse Scattering Transform, SIAM, 1980.

3. Andersen, H. C., Ratlle: a 'velocity' version of the shake algorithm for molecular dy. namics calculations, J. Comp. Phys. 52 (1983), 24-34.

4. Arakawa, A., Computational design for long-term numerical integration of the equations of tluid motion: Two dimensional incompressible flow. J. Comp. Phys. 1 (1966), 119-143.

5. Arakawa, A., A potential enstrophy and energy conserving scheme for the shallow water equations, Morithly Weacher Review 109 (1981), 18-36.

6. Arnol'd, V. I., Instability of dynamical systems with many degrees of freedom, Sov. Math. Dokl. 5 (1964), 581-584.

7. Arnol'd, V. I., and B. A. Khesin, Topological methods in hydrodynamics, Ann. Rev. Fluid Mech. 24 (1992), 145-166.

8. Benzel, S., Z. Ge, and C. Scovel, Elementary construction of higher order Lie-Poisson integrators, Phys. Lett. A 174 (1993), 229-232.

9. Benzel, S., McLachlan, R. I., and Scovel, C., Symplectic integration of the Euler fluid equations, in preparation.

10. Bochev, P., and C. Scovel, On quadratic invariants and symplectic structure, submitted to $B I T, 1993$.

11. Bourbaki, Lie Groups and Lie Algebras, Chapters 1-9, Springer-Verlag, New York, 1989.

12. Buttke, T. F. (1992) Lagrangian numerical methods which preserve the Hamiltonian structure of incompressible fluid flow, Vortex Flows and Related Numerical Methods, eds. Beale, J. T., Cottet, G.-H., and S. Huberson, Kluwer, Dordrecht, 1993.

13. Calvo, M. P. and J. M. Sanz-Serna, The development of variable-step symplectic integrators, with application to the two-body problem, SIAM J. Sci. Comp. 14 (1993), 936-952.

14. Cantrijn, F., Symplectic approach to nonconservative mechanics, J. Math. Phys. 25 (1984), 271-276.

15. Carineña and Ranada, Poisson maps and canonoid transformations for time-dependent Hamiltonian systems, J. Math. Phys. 30 (1989), 2258-2266.

16. Channell, P. J., The moment approach to charged particle beam dynamics, IEEE Trans. Nucl. Sci. NS-30 (1983), 2607-2609.

17. Channell, P. J., Explicit integration of kick Hamiltonians in three degrees of freedom, Los Alamos National Laboratory internal report AT-6: ATN-86-6, 1986.

18. Channell, P. J., and Scovel, J. C., Symplectic integration of Hamiltonian systems, Nonlinearity 3 (1990), 231-259.

19. Channell, P. J., and Scovel, J. C. Integrators for Lie-Poisson dynamical systems, Physica D 50 (1991), 80-88.

20. Cooper, G. J., Stability of Runge-Kutta Methods for Trajectory Problems, IMA J. Num. Anal. 7 (1987), 1-13.

21. Coste, A., P. Dazord, and A. Weinstein, Groupoides symplectiques, Publications $d u$ Departement de Mathematiques, Universite Claude Bernurd-Lyon I 2A (1987), 1-62. 
22. Cullen, M. J. P., J. Norbury, and R. J. Purser, Generalized Lagrangian solutions for atmospheric and oceanic flows, SIAM J. Appl. Math. 51 (1991), 20-31.

23. Dieci, L., Russell, R. D., and Van Vleck, E. S., Unitary integrators and applications to orthonormalization techniques, preprint, 1993.

2.4. Dodzuik, J., Finite difference approach to Hodge theory of harmonic forms, .4 m. J. Math. 08, 79-104.

25. Dowker, J. S., and Wolski, A., Finite model of two-dinensional ideal hydrodynanics, Phys. Rev. A 46 (1992) 6417-6430.

26. Dubrovin, B. A., Theory of operators and real algebraic geometry, Lect. Notes Math. 1334, 42-59.

27. Dubrovin, B. A., Differential-geometric Poisson brackets on a lattice, Funct. Anal. Appl. 23(2), 1989.

28. Earn, D. J. D., and S. Tremaine, Exact numerical studies of Hamiltonian maps: iterating without roundoff error, Physica $D 50$ (1992), 1-***

29. Eirola, T., and J. M. Sanz-Serna, Conservation of integrals and symplectic structure in the integration of differential equations by multistep methods, Numer. Math. 01 (1992), 281-290.

30. Fairlie, Fletcher, P. and Zachos, C. K. (1990) Infinite-dimensional algebras and a trigonometric basis for the classical Lie algebras, J. Math. Phys. 31(5) 1088-1094.

31. Feng, K., Difference scheme for Hamiltonian formalism and symplectic geometry, $J$. Comput. Math. 4 (1986), 279-289.

32. Feng. K., and M.-Z. Qin, The symplectic methods for the computation of Hamiltonian equations, in Numerical Methods for partial differential equations, Lect. Notes Math. 1297, Springer, Berlin New York, 1987, 1-31.

33. Fischer, H.-C., Automatic differentiation and applications, Math. Sci. Eng. 189 (1992), 105.

34. Fletcher, P. (1990), The Moyal bracket, in Quantum Groups (1990) pp. 143-157, World Scientific, Teaneck NJ, 1991.

35. Fomenko, A. T., and V. V. Trofimov, Integrable Systems on Lie Algebras and Symmetric Spaces, Gordon and Breach, New York, 1988.

36. Forest, E.., J. Bengtsson, and M. F. Reusch, Application of the Yoshida-Ruth techniques to implicit integration and multi-map explicit integration, Phys. Lett. A 158 (1991), 99-101.

37. Ge, Z., and $K$. Feng, On the approximation of linear Hamiltonian systents, J. Comp. Math. 6 (1988), 88-97.

38. Ge, $Z$., Equivariant symplectic difference schemes and generating functions, Physica $D$ 49 (1991), 376-386.

39. Ge, Z., Generating functions, Harnilton-Jacobi equations and symplectic groupoids on Poisson manifolds, Indiana Univ. Math. Journal 39:3 (1990), 859-876.

40. Ge, Z., On multiple-level symplectic integrators for Hamiltonian systems, preprint 1993.

41. Ge, Z., Kruse, H.-P., Marsden, J., and C. Scovel, The Shallow Water Approximation and Deformation of Poisson Brackets, in preparation.

42. Ge, Z., and J. Marsden, Lie-Poisson Hamilton-Jacobi theory and Lie-Poisson integrators, Phys. Lett. A 133 (1988), 135-139.

43. Ge, Z., and C. Scovel, Hamiltonian truncation of the shallow water equation, to appear in Lett. Math. Phys.

44. Glasner, M., Yevick, D., and Hermansson, B., Generalized propagation techniques for longitudinally varying refractive index distributions, Mathl. Comput. Modelling 16 (1992), 177-182.

45. Goldman, D., and T. J. Kaper, Third order operator splitting schemes and nonreversible systems, Los Alamos internal report LAUR-93-2781, 1993.

46. Grmela, M., Bracket formulation of diffusion-convection equations, Physica $D 21$ (1986), 179-212.

47. Hairer, E., Murua, A. and Sanz-Serna, J. M., The non-existence of symplectic multiderivative Runge-Kutta methods, preprint, 1993.

48. Holm. D. D., and Y. Kimura, Zero-helicity Lagrangian kinematics of three dimensional advection, Phys. Fluids A 3,(1991), 1033-1038. 
49. Holn, D. D., Y. Kimura, and C. Scovel, Lagrangian particle kinematics in 3-D convection, in Nonlinear Structures in Physical Systems, eds. L. Lam and 11. C. Morris, Springer, New York, 1990. **mp."***

50. Holm, D. D., Kuperschmidt, B. A., and C. D. Levermore. Hamiltonian differencing of Huid dynamics. Adv. Appl. Math. 6 (1985), 52-84.

51. Hoppe, J. (1989) Diffeomorphism groups, quantization, and $S(i(\infty)$, Int. J. Mod. Phys. A 4(19) 5235-5248.

52. Hoppe, J. (1990) Infunite dimensional algebras and $2+1$ dimensional field theory. Yet another view of $g l(\infty)$. Some new algebras. Rev. Math. Phys. 2(2) 193-200.

53. Hori, G., Theory of general perturbations with unspecified canonical variables, Publ. Astron. Soc. Japan 18 (1966), 287-296.

54. Hyman, J. M., and J. C. Scovel, Deriving mimetic difference approximations to differential operators using algebraic topology, Los Alamos National Laboratory Report (1988).

55. Hyman, J. M., Kaula, W. M., Newman, W. I., and C. Scovel, Integration schemes for n-body simulations, these conference proceedings.

56. Hyman, J. M., Knapp, R. J., and J. C. Scovel, High order finite volume approximations of differential operators on nonuniform grids, Physica $D 60$ (1992), 112-138.

57. Kraichnan, R. H., and D. Montgomery, Two-dimensional turbulence, Rep. Prog. Phys. $43(1980), 547-619$

is. Iserles, A., Bull. Cireek Math. Soc. 32 (1991), 3-20.

59. Isichenks, M. B., Can computer simulation predict the real behavior of turbulence?. Institute for Fusion Studies report 600, 1993.

(30. Jay, L., Symplectic partitioned Runge-Kutta methods for constrained Hamiltonian systems, Univ. de Genève preprint, 1993.

61. Kahaner, D. K., Automatic differentiation comments from Dr. Andreas Griewank, Sci. Inf. Bull. 17(4) (1992), 79.

62. Kaneko, K., Symplectic Cellular Automata, Phys. Lett. A 129 (1988), 9-16.

63. Karney, C. F. F, Numerical Techniques for the Study of Long Time Correlations, Particle Accelerators 19(1986), 213-221.

64. Kirchgraber, U., F. Lasagni, K. Nipp, and D. Stoffer, On the application of invariant manifold theory, in particular to numerical analysis, Int. Ser. Num. Math. 97 (1991), 189-197.

65. Koseleft, P.-V., Relations annong lie formal series and construction of symplectic integralors, preprint, 1992.

66. Leimkuhler, B., and R. D. Skeel, Symplectic numerical integrators in constrained Haniltonian systems, preprint, 1993.

67. Lewis, D. and J. Marsden, A Hamiltonian-dissipative decomposition of normal forms of vector fields, in Proc. Int. Conf. Bifurcation Theory and its Numerical Analysis, eds. K. Li, J. Marsden, M. Golubitsky, M., and G. Looss, Xian Jiaotong University Press, 1989.

68. Liao, G., P. Trong-Whay, and J. Su, A numerical grid generator based on Moser's deformation method, preprint, 1992.

69. Libermann, P., and C.-M. Marle, Symplectic Geometry and Analytical Mechanics, D. Reidel, Dordrecht, 1987.

70. MacEvoy, W., and C. Scovel, Integrators with variable stepsize for Hamiltonian systems, submitted to J. Comp. Appl. Math., 1993.

71. MacKay, R., Some aspects of the dynamics and numerics of Hamiltonian systems, in The Dynamics of Numerics and the Numerics of Dynamics, eds. D. S. Broomhead and A. Iserles, Oxford, 1992.

72. Mackenzie, K., Lie Groupoids and Lie Algebroids in Differential Geometry, LMS Lecture Notes Series 124, Cambridge University Press, 1987.

73. Maeda, S., Lagrangian formulation of discrete systems and concept of difference space, Math. Jeponica 37 (1982), 345-356.

74. Marsden, J., and A. Weinstein, Coadjoint orbits, vortices and Clebsch variables for incompressible fluids Physica 7D 1983 305-323

75. Marsden, J., and Ratiu, T., An Introduction to Mechanics and Symmetry (1992), preprint.

76. McLachlan, R. I. (1993) Explicit Lie-Poisson integration and the Euler equations, submitted to Phys. Rev. Lett. 
77. McLachlan, R. I. (1993) Symplectic integration of Hamiltonian wave equations, Numerische Mathematik (to appear).

78. McLachlan, R. I., On the numerical integration of ordinary differential equations by synumetric composition methods (1993), submitted to SIAM J. Sci. Comp.

79. McLachlan, R. I., and Atela, P., The accuracy of symplectic integrators, (1991) Nonlinearity 5, 541-562.

80. McLachlan, R. I., and C. Scovel, Equivariant constrained symplectic integration, sub. to Nonlinearity, 1993.

81. Mielke, A., Hamiltonian and Lagrangian Flows on Center Manifolds, Lect. Notes in Math. 1489, Springer Verlag, Berlin, 1991.

82. Miller, J., Weichman, P. B., and Cross, M. C. (1992), Statistical mechanics, Euler's equations, and Jupiter's red spot, Phys. Rev. A 45(4) 2328-2359.

83. Miller, R. H., Stellar dynamics, preprint, 1993.

84. Mokhov, O. I. (1989) Vorticity equation of two-dimensional hydrodynamics of an incompressible fluid as a canonical Hamiltonian system, Phys. Lett. A 139 363-368.

85. Morrison. P. J., Bracket formulation for irreversible classical fields, Phys. Lett. A 100 (1984), 423-427.

86. Morrison. P. J., A paradigm for joined Hamiltonian and dissipative systems, Physica $D$ 18 (1986), 410-419.

87. Moser, J., and Veselov, A. P., Discrete versions of some classical integrable systems and factorization of matrix polynomials, Commun. Math. Phys. 139 (1991), 217-243.

88. Moser, J. (1965), On the volume elements on a manifold Trans. Am. Math. Soc. 120 (1965), 286-294.

89. Neishtadt, A. I., The separation of motions in systems with rapidly rotating phase, $J$. Appl. Math. Mech. 48 (1984), 133-139,

90. Olver, P. (1988): Darboux' theorem for Hamiltonian differential operators, J. Diff. Eq. 71(1), 10-33

91. Olver, P. J., Applications of Lie groups to Differential Equations, 2nd ed., SpringerVerlag, New York, 1993.

92. Osedelets, V. I. (1989) On a new way of writing the Navier-Stokes equation. The Hamiltonian formalism. Comm. Moscow Math. Soc.

93. Nosé, S., A unified formulation of the constant temperature molecular dynamics methods, J. Chem. Phys. 81 (1984), 511-519.

94. Polychronakos, A. P. (1990) Aspects of $q$-Virasoro algebra, in Quantum Groups (1990), World Scientific, Teaneck NJ, 1991.

95. Qin, M.-Z., and W.-J. Zhu. Volume-preserving schemes and numerical experiments, Comp. Math. Appl. 26 (1993), 33-42.

96. Ramani, A., B. Grammaticos, and T. Bountis, Phys. Rep. 180(3) (1989), 159-245.

97. Ranuou, F., Numerical study of discrete plane area-preserving mappings, Astron. As. trophys. 31 (1974), 289-301.

98. Reich, S., Symplectic integration of constrained Hamiltonian systems by Runge-Kutta methods, Tech. report 93-13, Univ. British Columbia, 1993.

99. Reich, S., Momentum preserving symplectic integrators, preprint, 1993.

100. Reich, S., Numerical integration of the generalized Euler equations, preprint, 1993.

101. Reutenauer, C., Free Lie Algebras, Clarendon Press, Oxford, 1993.

102. Roberts, J. A. G., and G. R. W. Quispel, Chaos and time-reversal symmetry: order and chaos in reversible dynamical systems, Institu ut voor Theoretische Fysica Report 91-32, Universiteit van Amsterdam, Valckenierstraat 65, 1018 XE Amsterdam, The Netherlands, 1991.

103. Rouhi, A. and Arbarbanel, H. D. I. (1992) Symmetric truncations of the shallow water equations, preprint.

104. Saffman, P. G., Vortex Motion, Cambridge University Press, Cambridge, 1993.

105. Saha, P., and S. Tremaine, Symplectic integrators for solar system dynamics, Astron. J. 104 (1992), 1633-1640.

106. Saito, S., H. Sugiura, and T. Mitsui, Family of symplectic implicit Runge-Kutta formulae, BIT 32 (1992), 539-543.

107. Salmon, Rick, Semigeostrophic theory as a Dirac-bracket projection, J. Fluid Mech. 196 (1988), 345-358.

108. Salmon, R., Hamiltonian fluid mechanics, Ann. Rev. Fluid. Mech. 20 (1988), 225-256. 
109. Sanz-Serna, J. M., Runge-Kutta schemes for Hamiltonian systems, BIT 28 (1988), 877883.

110. Sanz-Serna, J. M., Symplectic integrators for Hamiltonian problems: An overview, in Acta Numerica 1992, pp. 243-286, Cambridge University Press, Cambridge, NY, 1992.

111. Scovel, J. C., Symplectic numerical integration of Hamiltonian systems, in The Geometry of Hamiltonian Systems, pp. 463-496, ed. Tudor Ratiu, MSRI Publ. 22, Springer-Verlag, New York, 1991.

112. Scovel, C., On symplectic lattice maps, Phys. Lett. A 159 (1991), 396-400.

113. Scovel, C., and A. Weinstein, Finite dimensional Lic-Poisson approximations to VlasovPoisson equations, to appear in Comm. Pure. Appl. Math., 1993.

114. Sheng, Q. (1989), Solving linear partial differential equations by exponential splitting, IMA J. Num. Anal. 9 199-212.

115. Simó, C., personal communication, 1992.

116. Skeel, R. D., personal communication, 1993.

117. Skeel, R. D., and C. W. Gear, Does variable stepsize ruin a symplectic integrator?, Physica D 60 (1992), 311-313.

118. Skeel, R. D., and J. J. Biesiadecki, Symplectic integration with variable stepsize, preprint, 1993.

119. Sleeman, B. D., Complexity in biological systems and Hamiltonian dynamics, Proc. $R$. Soc. Lond. A 425 (1989), 17-47.

120. Stofer, D. M., Some geometric and numerical methods for perturbed integrable systems, Thesis, ETH-Zürich, 1988.

121. Suris, Yu. B., Integtable mappings of the standard type, Funct. Anal. Appl. 23(1) (1989), $74-75$.

122. Suzuki, M., Fractal decomposition of exponential operators with applications to manybody theories and Monte-Carlo simulations, Phys. Lett. A 146 (1990), 319-323.

123. Suzuki, M., Symplectic decomposition theory of time evolution operators in nonseparable Hamiltonian systems, preprint, 1993.

124. Symes, W. W., The $Q R$ algorithm and scattering for the finite nonperiodic Toda lattice, Physica D 4 (1982) 272-280.

125. Tang, Yi-Fa (1993), The symplecticity of multi-step methods, Comp. Math. Appl. 25 83-90.

126. Virasoro, M. A., Variational principle for two-dimensional incompressible hydrodynamics and quasigeostrophic flows, Phys. Rev. Lett. 47 (1981) 1181-1183.

127. Vujanovic, B. D., and S. E. Jones, Variational Mcthods in Nonconservative Phenomena Academic Press, Boston, 1989.

128. Weinstein A., The local structure of Poisson manifolds, J. Diff. Geom. 18 (1983), 523557.

129. Winckelmans, G. S. and Leonard, A., Contributions to vortex particle methods for the computation of three-dimensional unsteady flows, preprint, 1993.

130. Wisdom and $M$. Holman (1991), Symplectic maps for the $N$-body problem Astron. J. 102(4) 1528-1538; II: stability analysis, Astron. J. 104(5) 2022-2029.

131. Yoshida, H., Recent progress in the theory and application of symplectic integrators, Cel. Mech. Dyn. Astr. 56 (1993), 27-43.

132. Zakharov, V. E. (1990), The algebra of integrals of motion of two-dimensional hydrodynamics in Clebsch variables, Funct. Anal. Appl. 189-196.

133. Zeitlin, V., Finite-mode analogues of $2 \mathrm{D}$ ideal hydrodynamics: Coadjoint orbits and local canonical structure, Physica D 49 (1991), 353-362. 

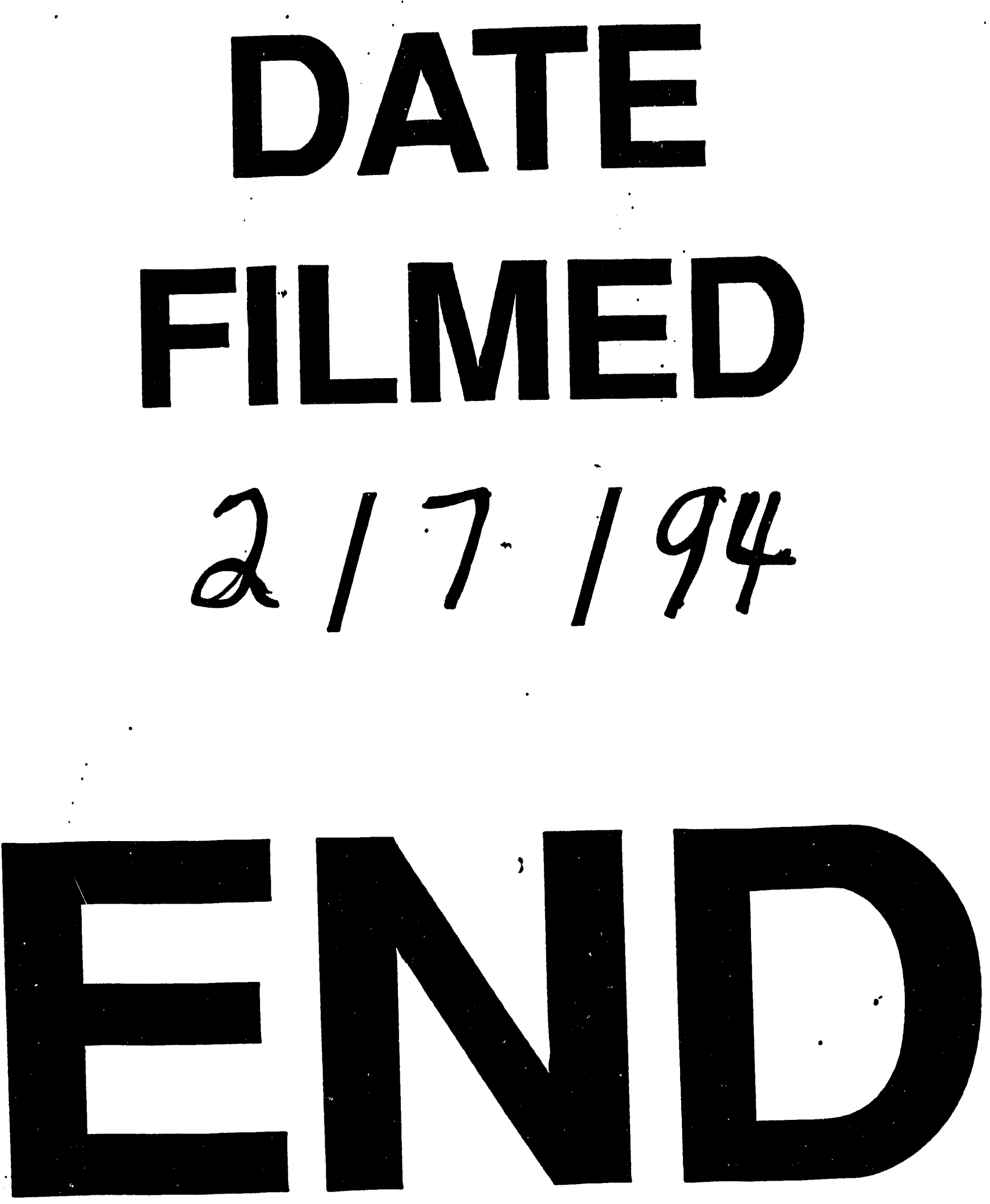


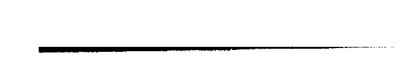

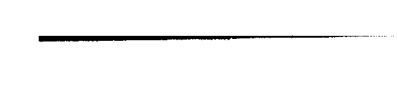

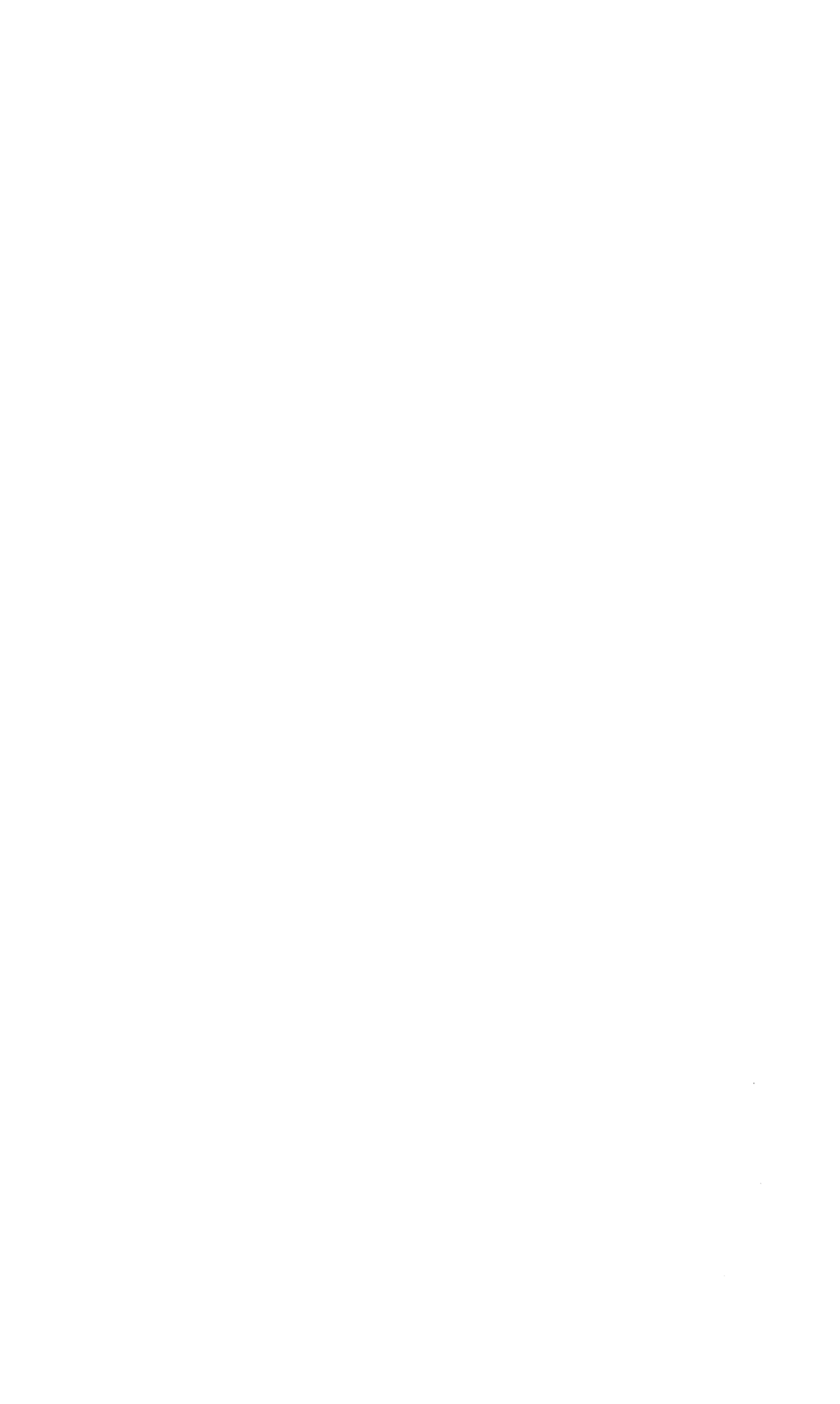

